\title{
Different levels of cardiometabolic indicators in multiple vs. singleton children
}

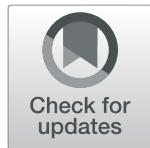

Maria João Fonseca ${ }^{1 *}$ (D), Ana Cristina Santos ${ }^{1,2}$ and Henrique Barros ${ }^{1,2}$

\begin{abstract}
Background: We aimed to compare cardiometabolic indicators in singletons and multiples at age 7 and explore the birthweight mediation effect.

Methods: We studied 5431 singletons and 103 sets of multiples from Generation XXI birth cohort. Anthropometric measurements, body composition, and fasting blood samples were obtained. Age- and sex-specific z-scores were calculated (additionally height-specific for blood pressure). Adjusted regression coefficients and respective 95\% confidence intervals $[\beta(95 \% \mathrm{Cl})]$ were computed using path analysis.

Results: Multiples had lower weight $[-0.419(-0.616 ;-0.223)]$, height $[-0.404(-0.594 ;-0.213)]$, BMI $[-0.470$ $(-0.705 ;-0.234)]$, fat mass index $[-0.359(-0.565 ;-0.152)]$, waist circumference $[-0.342(-0.537 ;-0.147)]$, and waist-to-height ratio $[-0.165(-0.326 ;-0.003)]$ z-scores. These results were explained by the indirect effect via birthweight, which was also negative and significant for all the aforementioned cardiometabolic indicators, while no direct effect was present. There were also significant indirect effects regarding fat-free mass index, glucose, insulin, and blood pressure, though the total effects were not significant, due to the balance between direct and indirect effects. The only significant direct effect was regarding diastolic blood pressure $[-0.165(-0.302 ;-0.028)]$.

Conclusions: At age 7, multiples presented better cardiometabolic indicators explained by lower weight at birth, except for the lower blood pressure which was independent of an effect via birthweight.
\end{abstract}

Keywords: Anthropometric measures, Birthweight, Body composition, Cardiometabolic, Twins

\section{Background}

An offspring of a multiple pregnancy differs from a singleton regarding characteristics such as fetal growth $[1,2]$, gestational age $[1,3]$, mode of delivery $[3,4]$, anthropometric measures at birth $[1,3,4]$ and complications during the neonatal period $[1,4]$.

Usually, multiples present a lower birthweight, due to intrauterine growth restriction $[1,2]$ and lower gestational age $[1,3]$. Low birthweight is associated with increased risk of neonatal morbidity and mortality, but also with adverse long-term consequences, namely those related with early cardiometabolic programming [5]. Yet, studies performed in singletons showed that either low or high birthweight are associated with obesity and other unfavourable cardiometabolic markers [5-8]. Moreover,

\footnotetext{
* Correspondence: mjoao.fonseca@ispup.up.pt

${ }^{1}$ ISPUP-EPIUnit, Universidade do Porto, Rua das Taipas nº 135, 4050-600

Porto, Portugal
}

Full list of author information is available at the end of the article the particular intrauterine growth circumstances of multiples might impact on later health [2]. Considering neonatal prognostic, optimal birthweight seems to be lower for multiple babies than for singletons [9], yet this effect seems to disappear when adjusted for relative birthweight [10]. The same may not hold true for long-term outcomes.

In addition to a slower rate of growth both in utero $[1,2]$ and during the first years of life [11, 12], multiple babies experience particular circumstances [13] (e.g.: sharing the utero and nutrition during intra uterine life, sharing the mother's and father's attention, having a same-age brother/ sister), which might be influential throughout life.

Thus, we can hypothesize that multiples conserve particular anthropometric parameters during childhood and different levels of cardiometabolic indicators. Yet, to the best of our knowledge, no study has so far evaluated the effect of being multiple on cardiometabolic indicators

(c) The Author(s). 2019 Open Access This article is distributed under the terms of the Creative Commons Attribution 4.0 International License (http://creativecommons.org/licenses/by/4.0/), which permits unrestricted use, distribution, and 
during childhood. Thus, we compared anthropometric, body composition, serum cardiometabolic markers, and blood pressure in multiples and singletons at 7 years of age and explored the mediation effect of birthweight.

\section{Methods}

Participants of the present study are part of the Generation XXI birth cohort [14]. Between April 2005 and August 2006, mothers given birth at one of the five level III public units providing obstetrical and neonatal care in the metropolitan area of Porto, Portugal, were consecutively invited to participate. All the maternities, except one, were included in a general hospital, with a variety of medical and surgical specialties, and all corresponded to level III maternity units, with differentiated perinatal support. At birth, $91.4 \%$ of the invited mothers accepted to participate. A total of 8495 mothers, who gave birth to 8647 live born infants, which included 137 twin pairs, 6 triplets, 1 quadruplet, and 8351 singletons, were enrolled into the cohort. At 7 years of age, all cohort members were invited to be re-evaluated (April 2012 to March 2014), and 6889 (80\%) agreed to that follow-up.

At recruitment, information on maternal socio-demographic, behavioural and pre-pregnancy anthropometric characteristics was collected by face-to-face interview using structured questionnaires, completed 24 to $72 \mathrm{~h}$ after delivery, during the hospital stay. Data on delivery and newborn characteristics were additionally abstracted from clinical records, as previously described $[7,14]$.

At 7 years of age, child's anthropometric measurements were performed by trained examiners, according to standard procedures, as previously described [7]. Waist-to-height ratio (WHtR) was calculated as WHtR = Waist Circumference $(\mathrm{WC})(\mathrm{cm}) /$ height $(\mathrm{cm})$. Child's Body Mass Index (BMI) was calculated as $\mathrm{BMI}=$ weight $(\mathrm{kg}) /$ height $^{2}(\mathrm{~m})$ and age- and sex-specific z-scores were established according to the World Health Organization [15]. Body composition was measured by a bioelectrical impedance analysis generator [7] and following the approach proposed by Horlick et al. [16], we previously tested all equations considered by the author, and chose the one proposed by Schaefer et al. [17]. Fat mass (FMI) and fat-free mass indexes (FFMI) were calculated as FMI = total fat $(\mathrm{kg}) /$ height $^{2}(\mathrm{~m})$ and $\mathrm{FFMI}=$ total fat-free mass $(\mathrm{kg}) /$ height $^{2}(\mathrm{~m})$. Age- and sex-specific $\mathrm{z}$-scores were established for weight, height, WC, WHtR, FMI and FFMI based on the age- and sex-specific means and standard deviations (SD) derived from the whole cohort.

Two measurements of blood pressure, separated by at least $5 \mathrm{~min}$, were taken after 10 -min rest. If the difference between the two individual measurements was lower than $5 \mathrm{mmHg}$, the mean was recorded, if larger than $5 \mathrm{mmHg}$ a third measurement was taken and the mean of the 2 closest values was used. For systolic (SBP) and diastolic (DPB) blood pressure, age-, sexand height-specific z-scores were calculated, following the recommendations of the American Academy of Pediatrics [18].

After an overnight fast, a venous blood sample was drawn, after applying a topical analgesic cream (EMLA cream), centrifuged at $3500 \mathrm{rpm}$ for $10 \mathrm{~min}$, aliquoted, immediately analysed or deep frozen until analysis. Glucose was measured using an UV enzymatic assay (hexokinase method), insulin using electrochemiluminescence immunoassay, HDL-cholesterol and triglycerides (TG) using enzymatic colorimetric assays, and high-sensitivity C-reactive protein (hs-CRP) using nephelometry. Ageand sex-specific z-scores were computed based on the age- and sex-specific means and standard deviations (SD) derived from the whole Generation XXI cohort.

The cardiometabolic traits analyzed included anthropometrics, body composition, serum cardiometabolic markers, and blood pressure to be as much comprehensive as possible. Concerning serum cardiometabolic markers we chose to include those present in the definition of the metabolic syndrome (glucose, TG, and HDL-cholesterol) and added insulin and hs-CRP as extra markers of glucose metabolism and inflammatory process, respectively.

At 7 years of age, 212 multiples and 5431 singletons had a physical exam and provided blood samples. The comparison between these participants and the remaining eligible children, stratified by singleton/multiple status, is shown in Table 1. Mothers of singleton participants were older, more educated, more frequently were married or living with a partner, were primiparae, had been more frequently submitted to fertility treatment, had a higher prevalence of caesarean delivery, and less frequently smoked during the third pregnancy trimester. Among multiples, participant mothers were more educated, the babies presented higher birthweight, and a higher frequency of monozygotic twins.

All the phases of the study complied with the Ethical Principles for Medical Research Involving Human Subjects expressed in the Declaration of Helsinki. The study was approved by the University of Porto Medical School/ S. João Hospital Centre Ethics Committee and parents or legal representatives signed an informed consent. The study was approved by the Portuguese Data Protection Authority.

\section{Statistical analysis}

Proportions were compared using the chi-square test or Fisher exact test whenever adequate, and means were compared using student t-test (analysis performed using SPSS version 23.0). 
Table 1 Maternal, pregnancy, delivery and newborn characteristics of participants and eligible non participants, stratified by singleton/multiple status

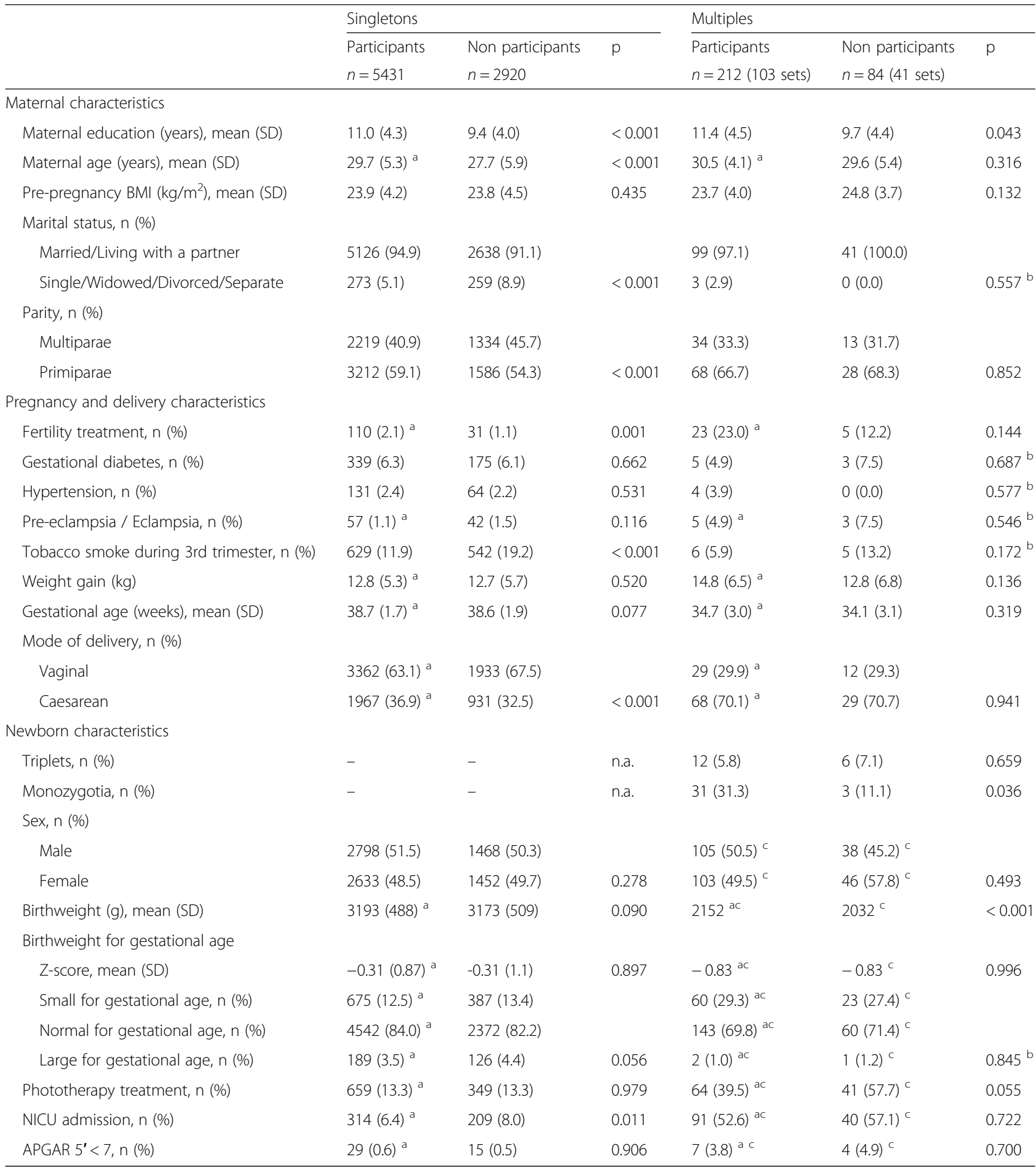

${ }^{a}$ Significant differences $(p<0.05)$ between singleton participants and multiple participants

${ }^{\mathrm{b}}$ Fisher exact test

${ }^{\mathrm{C}}$ Adjusted for non independence

Linear regression coefficients $(\beta)$ and $95 \%$ confidence intervals $(95 \% \mathrm{CI})$ were computed using path analysis. Since this method does not allow adjustment for non- independence, a random child was chosen from each set of twins to perform the path analysis. The fit of the models was assessed using different indexes: the 
Comparative Fit Index (CFI), the Tucker-Lewis Index (TLI), and the Root Mean Square Error of Approximation (RMSEA). A good model fit is indicated by a CFI and TLI values $\geq 0.90$ and values of RMSEA lower than 0.05 .

We defined confounders as events occurring prior to conception that were associated with both singleton/ multiple status and the outcomes and mediators as postconception events that were associated both with singleton/multiple status and the outcomes, being an intermediate step in the hypothesized causal chain [7]. Maternal age, education, pre-pregnancy BMI, marital status, parity, and fertility treatment were tested as potential confounders. Diabetes and hypertension during pregnancy, pre-eclampsia/eclampsia, gestational age, mode of delivery, birthweight, and birthweight for gestational age [19] were tested as potential mediators. Of those variables, only maternal age, fertility treatment, gestational age, birthweight, and birthweight for gestational age were significantly associated with both singleton/multiple status and at least one of the outcomes. We found strong correlations between gestational age, birthweight, and birthweight for gestational age. However, of these three potential mediators, birthweight presented the strongest association with the outcomes. Also, interactions with birthweight, sex and mode of delivery were tested and not found.

Accordingly, the confounders included in the models were maternal age (years) and fertility treatment (yes vs. no) and the mediator included was birthweight (decigram). Path analysis was performed for each of the outcomes (z-scores) with Mplus on R software [20]; 95\% confidence intervals were calculated by bootstrapping.

\section{Results}

Mean maternal age (30.5 vs. 29.7 years, $p=0.043)$ and the frequency of fertility treatment $(23.0 \%$ vs. $2.1 \%, p<$ 0.001 ) were higher among multiples (Table 1). Considering the potential mediators, multiples had higher proportion of pre-eclampsia/eclampsia during pregnancy ( $4.9 \%$ vs. $1.1 \%, p=0.006)$, lower mean gestational age (34.7 vs. 38.7 weeks, $p<0.001$ ), higher proportion of caesarean delivery $(70.1 \%$ vs. $36.9 \%, p<0.001)$, lower mean birthweight (2152 g vs. $3193 \mathrm{~g}, p<0.001)$ and lower $\mathrm{z}$ scores of birthweight for gestational age $(-0.83$ vs. $0.31, p<0.001$ ) than singletons (Table 1 ).

As observed in Table 2, multiples had lower mean weight ( 24.0 vs. $26.2 \mathrm{~kg}, p<0.001)$, height (121.3 vs. $123.6 \mathrm{~cm}, p<0.001)$, BMI (16.3 vs. $\left.17.1 \mathrm{~kg} / \mathrm{m}^{2}, p<0.001\right)$, FMI ( 2.3 vs. $\left.3.0 \mathrm{~kg} / \mathrm{m}^{2}, p=0.001\right)$, WC (56.6 vs. $59.1 \mathrm{~cm}$, $p<0.001)$, WHtR (0.47 vs. $0.48, p=0.023)$, SBP (103.4 vs. $105.2 \mathrm{mmHg}, p=0.016)$ and DBP (68.1 vs. 69.8 $\mathrm{mmHg}, p=0.006$ ) than singletons. When comparing the $\mathrm{z}$-scores of the aforementioned cardiometabolic indicators, the results were similar, except for SBP and DBP zscores, which were similar in singletons and multiples. As a sensitivity analysis, the same comparison was performed restricting the analysis only to small for gestational age children (Additional file 1: Table S1) and the results were similar. Small for gestational age multiples had significantly lower mean BMI, FFMI, WC, WHtR, SBP and DBP than small for gestational age singletons.

Table 2 Comparison between singletons and multiples regarding cardiometabolic indicators at 7 years follow-up evaluation

\begin{tabular}{|c|c|c|c|c|c|c|}
\hline & \multicolumn{2}{|l|}{ Crude value } & \multirow[t]{2}{*}{$\mathrm{p}$} & \multicolumn{2}{|l|}{ Age and sex z-score ${ }^{b}$} & \multirow[t]{2}{*}{$p$} \\
\hline & Singletons & Multiples & & Singletons & Multiples & \\
\hline \multicolumn{7}{|c|}{ Cardiometabolic characteristics at age $7^{a}$} \\
\hline Weight (kg) & $26.2(26.1,26.4)$ & $24.0(23.1,25.0)$ & $<0.001$ & $0.02(-0.01 ; 0.04)$ & $-0.41(-0.59 ;-0.24)$ & $<0.00$ \\
\hline Height $(\mathrm{cm})$ & $123.6(123.4,123.7)$ & $121.3(120.3,122.2)$ & $<0.001$ & $-0.01(-0.04 ; 0.02)$ & $-0.45(-0.62 ;-0.28)$ & $<0.00$ \\
\hline Body mass index $\left(\mathrm{kg} / \mathrm{m}^{2}\right)$ & $17.1(17.0,17.1)$ & $16.3(15.8,16.7)$ & $<0.001$ & $0.73(0.70 ; 0.76)$ & $0.27(0.07 ; 0.48)$ & $<0.00$ \\
\hline Fat mass index $\left(\mathrm{kg} / \mathrm{m}^{2}\right)$ & $3.0(2.9,3.1)$ & $2.3(1.9,2.7)$ & 0.001 & $-0.07(-0.10 ;-0.04)$ & $-0.39(-0.57 ;-0.21)$ & 0.001 \\
\hline Fat-free mass index $\left(\mathrm{kg} / \mathrm{m}^{2}\right)$ & $14.0(14.0,14.1)$ & $13.9(13.7,14.2)$ & 0.360 & $0.09(0.07 ; 0.12)$ & $0.01(-0.16 ; 0.18)$ & 0.325 \\
\hline Waist circumference (cm) & $59.1(59.0,59.3)$ & $56.6(55.4,57.8)$ & $<0.001$ & $-0.03(-0.06 ;-0.01)$ & $-0.40(-0.57 ;-0.23)$ & $<0.00$ \\
\hline Waist-to-height ratio & $0.48(0.48,0.48)$ & $0.47(0.46,0.48)$ & 0.023 & $-0.05(-0.07 ;-0.03)$ & $-0.20(-0.34 ;-0.06)$ & 0.044 \\
\hline Glucose (mg/dL) & $82.4(82.2,82.7)$ & $82.4(80.7,84.1)$ & 0.980 & $-0.09(-0.13 ;-0.05)$ & $-0.11(-0.40 ; 0.19)$ & 0.909 \\
\hline Insulin ( $\mu \mid \mathrm{U} / \mathrm{mL})$ & $5.2(5.0,5.3)$ & $4.5(3.8,5.2)$ & 0.061 & $0.01(-0.03 ; 0.04)$ & $-0.20(-0.43 ; 0.03)$ & 0.082 \\
\hline HDL-cholesterol (mg/dL) & $56.3(55.9,56.7)$ & $58.0(55.4,60.6)$ & 0.201 & $0.08(0.04 ; 0.11)$ & $0.22(-0.01 ; 0.44)$ & 0.218 \\
\hline Triglycerides (mg/dL) & $62.2(61.3,63.2)$ & $60.9(54.8,67.1)$ & 0.693 & $-0.05(-0.09 ;-0.02)$ & $-0.09(-0.29 ; 0.12)$ & 0.745 \\
\hline hs-C-reactive protein (mg/L) & $1.31(1.20,1.43)$ & $1.02(0.43,1.61)$ & 0.346 & $0.00(-0.03 ; 0.04)$ & $-0.03(-0.28 ; 0.22)$ & 0.816 \\
\hline Systolic blood pressure (mmHg) & $105.2(105.0,105.5)$ & $103.4(102.0,104.9)$ & 0.016 & $0.71(0.68 ; 0.73)$ & $0.70(0.58 ; 0.83)$ & 0.970 \\
\hline Diastolic blood pressure $(\mathrm{mmHg})$ & $69.8(69.7,70.1)$ & $68.1(67.0,69.4)$ & 0.006 & $1.04(1.02 ; 1.06)$ & $0.97(0.86 ; 1.07)$ & 0.185 \\
\hline
\end{tabular}

${ }^{a}$ Mean values and $95 \%$ confidence intervals adjusted for non independence

${ }^{b}$ For systolic and diastolic blood pressure - age, sex and height $\mathrm{z}$-score 
Additionally, also as a sensitivity analysis, a matched pair analysis, matching each multiple with the singleton with the closest gestational age, was performed and all the results were in the same direction - multiples presented lower z-scores of weight, height, BMI, FMI, WC, and WHtR - significant only for weight, height and WC (Additional file 2: Table S2).

Table 3 shows the total, direct and indirect effect of multiple status on cardiometabolic indicators z-scores at 7 years of age, resulting from the path analysis. Multiples had lower age and sex $\mathrm{z}$-scores for weight $[-0.419$ ($0.616 ;-0.223)]$, height $[-0.404(-0.594 ;-0.213)]$, BMI $[-0.470(-0.705 ;-0.234)]$, FMI $[-0.359(-0.565 ;-$ $0.152)]$, WC $[-0.342(-0.537 ;-0.147)]$, and WHtR [$0.165(-0.326 ;-0.003)]$. These total effects were explained by the indirect effect via birthweight, which was also negative and significant for all the previous mentioned cardiometabolic indicators, while no direct effect was present. There were also significant indirect effects regarding FFMI, glucose, insulin, SBP and DBP, though the total effect was not significant, due to the balance between the direct and indirect effects. The only significant direct effect observed was for DBP [-0.165 ($0.302 ;-0.028)]$.

Figure 1 presents the regression coefficients and 95\% confidence intervals $[\beta(95 \% \mathrm{CI})]$ for the different paths of the associations. In this figure, the indirect effects can be observed, comprising an inverse association between multiple status and birthweight plus: a) a positive association between birthweight and weight, height, BMI, FMI, FFMI, WC, WHtR, glucose and insulin; b) an inverse association between birthweight and SBP and DBP.

\section{Discussion}

In this study, we compared multiples and singletons regarding cardiometabolic indicators at age 7 . We found that multiples presented lower adiposity, with significantly lower adjusted weight, height, BMI, FMI, and central adiposity. These differences were explained by their lower birthweight. Multiples had also lower blood pressure, after removing the effect via birthweight. Multiples and singletons were similar regarding FFMI, glucose, insulin, HDL-cholesterol, triglycerides, and hs-CRP levels.

Multiples were shorter, lighter, and thinner than singletons. The lower BMI was largely explained by a lower FMI - 0.359 standard deviations lower in multiples. In addition, multiples had lower WC and WHtR, meaning less central adiposity. After removing the effect via birthweight, the observed associations were attenuated towards zero, no direct effect being present. In contrast, the indirect effect (via birthweight) was significant for all those variables, which means that multiples presented lower estimates for anthropometric and body composition measures reflecting mostly their lower birthweight. Multiples have lower birthweight [1-3], which in turn has been associated with lower anthropometric and body composition measurements later in life, in several recent

Table 3 Total, direct and indirect effect of multiple status (vs. singleton status) on cardiometabolic indicators z-scores at 7 years of age

\begin{tabular}{|c|c|c|c|}
\hline & \multicolumn{3}{|l|}{$\beta[95 \% \mathrm{Cl}]$ in Multiples ${ }^{a}$} \\
\hline & Total effect & Direct effect & Indirect effect \\
\hline \multicolumn{4}{|l|}{ Age and sex $z$-scores ${ }^{b}$} \\
\hline Weight & $-0.419(-0.616 ;-0.223)^{*}$ & $-0.020(-0.221 ; 0.181)$ & $-0.399(-0.466 ;-0.333)^{*}$ \\
\hline Height & $-0.404(-0.594 ;-0.213)^{*}$ & $0.083(-0.110 ; 0.275)$ & $-0.486(-0.556 ;-0.416)^{*}$ \\
\hline Body mass index & $-0.470(-0.705 ;-0.234)^{*}$ & $-0.133(-0.376 ; 0.109)$ & $-0.336(-0.409 ;-0.263)^{*}$ \\
\hline Fat mass index & $-0.359(-0.565 ;-0.152)^{*}$ & $-0.109(-0.322 ; 0.104)$ & $-0.249(-0.312 ;-0.187)^{*}$ \\
\hline Fat-free mass index & $-0.057(-0.259 ; 0.145)$ & $0.019(-0.192 ; 0.229)$ & $-0.075(-0.133 ;-0.018)^{*}$ \\
\hline Waist circumference & $-0.342(-0.537 ;-0.147)^{*}$ & $-0.086(-0.287 ; 0.115)$ & $-0.256(-0.315 ;-0.196)^{*}$ \\
\hline Waist-to-height ratio & $-0.165(-0.326 ;-0.003)^{*}$ & $-0.096(-0.264 ; 0.072)$ & $-0.069(-0.115 ;-0.023)^{*}$ \\
\hline Glucose & $0.218(-0.117 ; 0.553)$ & $0.300(-0.044 ; 0.644)$ & $-0.082(-0.160 ;-0.004)^{*}$ \\
\hline Insulin & $-0.141(-0.419 ; 0.137)$ & $-0.072(-0.358 ; 0.213)$ & $-0.069(-0.133 ;-0.004)^{*}$ \\
\hline HDL-cholesterol & $0.148(-0.112 ; 0.408)$ & $0.122(-0.145 ; 0.389)$ & $0.026(-0.034 ; 0.086)$ \\
\hline Triglycerides & $-0.014(-0.254 ; 0.226)$ & $-0.004(-0.250 ; 0.243)$ & $-0.010(-0.066 ; 0.045)$ \\
\hline hs-C-reactive protein & $-0.093(-0.374 ; 0.188)$ & $-0.107(-0.396 ; 0.182)$ & $0.014(-0.051 ; 0.079)$ \\
\hline Systolic blood pressure & $0.057(-0.103 ; 0.217)$ & $-0.056(-0.222 ; 0.110)$ & $0.113(0.066 ; 0.159)^{*}$ \\
\hline Diastolic blood pressure & $-0.064(-0.196 ; 0.068)$ & $-0.165(-0.302 ;-0.028)^{*}$ & $0.100(0.062 ; 0.139)^{*}$ \\
\hline
\end{tabular}




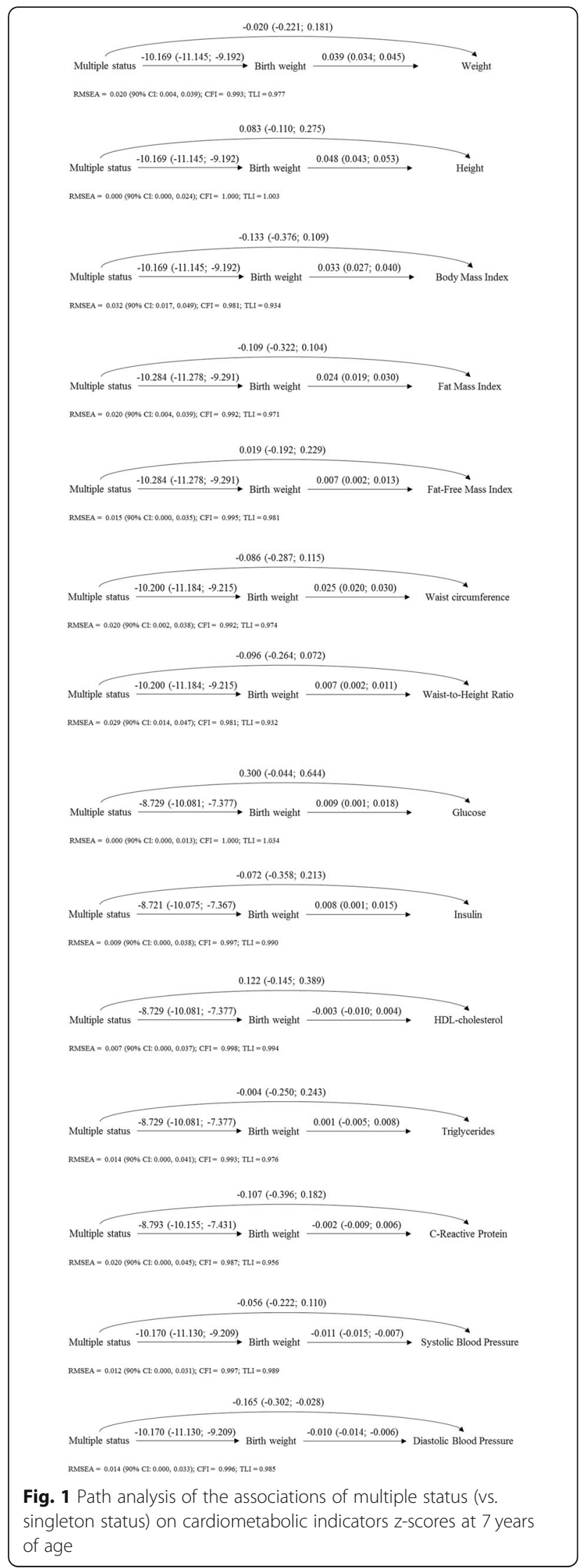

cohorts [6, 21, 22], including Generation XXI [7]. This is explained by the tracking effect, and in this regard, literature shows that BMI and fatness tracks from early life until adulthood [23, 24]. On the other hand, it is known that catch-up growth, the acceleration in growth that occurs when a period of growth retardation ends and favourable conditions are restored [25], usually occurs in children that are born with lower birthweight, which is the case of multiples. However, data from the third National Health and Nutrition Examination Survey (NHANES) and the National Collaborative Perinatal Project showed that despite the catch-up growth, children that were born with lower birthweight remained shorter and lighter through childhood [26, 27], which may explain why multiples remain smaller than singletons during childhood. Sensitivity analysis were performed taking into consideration other potential mediators and none fully explained the lower anthropometric and body composition measures, except for birthweight.

Concerning blood pressure, the levels of sex-, age- and height- specific z-scores were similar in multiples and singletons. Similarly, a study using data from the Netherlands Twin Registry found that multiples and their singleton siblings had similar blood pressure levels in adulthood [28]. Nevertheless, when the mediator effect via birthweight was removed, a direct association between multiple status and lower blood pressure emerged, significant for DBP. Lower birthweight has been described as a risk factor for higher blood pressure [29], this being partly explained by decreased number of nephrons in lower birthweight children $[29,30]$, suggesting that multiples could have higher blood pressure than singletons, merely due to their lower birthweight (i.e. a positive birthweight-mediated association, which actually occurred in the present study, since the indirect effect was positive and significant for SBP and DBP). However, when analyzing the association of singleton/multiple status with blood pressure independently of this birthweightmediated effect, multiple status was directly associated with lower blood pressure (i.e. for a given fixed birthweight, multiples would have lower blood pressure than singletons). We further tested adjustment for other possible mediators - current BMI and current weight - but none explained the association. So, other mechanisms than the attained weight must be involved in this direct association of multiple status and lower blood pressure. One of those mechanisms could be the hypothesis of in utero programming, where a different in utero development of kidneys or of hypothalamic-pituitary-adrenal-axis makes possible that for a given birthweight multiples present a lower blood pressure than singletons. It had already been shown in animal models that despite multiples do have a lower number of nephrons, they did not present higher blood pressure levels [30]. Our data needs 
to be replicated in different samples to clarify why multiples, despite having lower birthweight, do not present higher blood pressure later in life and may even have lower blood pressure when analyzing independently of birthweight.

At the age of 7, blood levels of glucose, insulin, HDLcholesterol, triglycerides, and hs-CRP were similar in multiples and singletons. Since multiplicity is relatively uncommon, it would be possible that these findings reflect lack of statistical power. However, given that most point estimates were close to the null, this does not seem probable.

\section{Limitations and strengths}

This study includes a population-based sample and is the first to examine the effect of singleton/multiple status on cardiometabolic indicators in childhood. Although statistically significant differences between participants and non-participants existed, the magnitude of those differences were small, not expecting to have influenced the conclusions.

At 7 years of age, information was obtained using measurements performed by trained health professionals according to standard procedures, which preclude recall bias. Although information on a 24-h blood pressure assessment were not available, we believe that the blood pressure measured on a single occasion was capable of discriminate children.

The low number of multiples, which is expected in a general population-based study, increases the chance of type II error. Nevertheless, associations between multiple status and anthropometric and body composition parameters and also blood pressure were detected. However, it is possible that type II error occurred when analyzing blood parameters.

Sensitivity analyses were performed - one restricting the analysis only to small for gestational age children and the other matching each multiple with the singleton with the closest gestational age (matched pair analysis) in order to explore, to some extent, the role of these two potential mediators. The results were in the same direction, although some lost statistical significance or were attenuated, which was expected because when we take into account gestational age or birthweight for gestational age, we are inevitably taking birthweight into account. Additionally, in the matched pair analysis, DBP appeared to be lower in multiples, which also occurred when we adjusted for birthweight. So, we believe these results improve the level of evidence of our main findings.

The present study is one of the few to evaluate cardiometabolic indicators this early in life. As abnormal values of cardiometabolic indicators are unusual at these ages, it is possible that the medium- and/or long-term effects of multiple/singleton status on cardiometabolic indicators are not yet fully evident and, with aging, stronger long-term effects might appear. Still, a multiple/ singleton status effect on anthropometric and body composition measures and blood pressure seemed evident.

Even though birthweight explained most of the association between multiple status and cardiometabolic traits, we are aware that other variables such as gestational age, immaturity or mode of delivery could also play a role. Yet, given the multicollinearity, when taking into account the mediator birthweight, we are also taking into account part of such variables.

Despite the limitations of the present study, we believe that our results demonstrate how multiples and singletons differ in terms of cardiometabolic indicators at 7 years of age and generate the hypothesis of an explanation via birthweight. Yet, these results should be replicated in other populations and other mechanisms explaining the differences found between multiples and singletons must be explored.

\section{Conclusions}

At age 7, multiples presented lower total and central adiposity explained by a lower weight at birth. Multiple status also affect glucose, insulin and SBP, but only indirectly via birthweight, i.e. multiples had lower birthweight which in turn led to lower glucose and insulin levels and higher SBP.

\section{Additional files}

Additional file 1: Table S1. Comparison between small for gestational age singletons and small for gestational age multiples regarding cardiometabolic indicators at 7 years follow-up evaluation. (DOCX $16 \mathrm{~kb}$ )

Additional file 2: Table S2. Matched pair analysis regarding the comparison between singletons and multiples regarding cardiometabolic indicators at 7 years follow-up evaluation. (DOCX $14 \mathrm{~kb}$ )

\section{Abbreviations}

BMI: body mass index; DBP: diastolic blood pressure; FFMI: fat-free mass index; FMI: Fat mass index; hs-CRP: high-sensitivity C-reactive protein; LDLC: low density lipoprotein cholesterol; SBP: systolic blood pressure; TG: triglycerides; WC: Waist Circumference; WHtR: Waist-to-height ratio

\section{Acknowledgements}

The authors gratefully acknowledge the families enrolled in Generation XXI for their kindness, all members of the research team for their enthusiasm and perseverance and the participating hospitals and their staff for their help and support.

\section{Authors' contributions}

MJF performed literature search, data collection, analysis and interpretation and was responsible for the first draft of the manuscript. ACS participated in funding acquisition and critically reviewed the manuscript. HB participated in funding acquisition, contributed to the study design and critically reviewed the manuscript. All authors read and approved the final manuscript.

\section{Funding}

Generation XXI was funded by Programa Operacional de Saúde - Saúde XXI, Quadro Comunitário de Apoio III and Administração Regional de Saúde 
Norte (Regional Department of Ministry of Health); FEDER through the Operational Programme Competitiveness and Internationalization and national funding from the Foundation for Science and Technology - FCT (Portuguese Ministry of Science, Technology and Higher Education) (POCl-010145-FEDER-016837), under the project "PathMOB: Risco cardiometabólico na infância: desde o início da vida ao fim da infância" (Ref. FCT PTDC/DTP-EPI/3306/ 2014), the Unidade de Investigação em Epidemiologia - Instituto de Saúde Pública da Universidade do Porto (EPIUnit) (POCI-01-0145-FEDER-006862; Ref. UID/DTP/ 04750/2013), and ACS holds a FCT Investigator contract IF/01060/2015; and by the project DOCnet (NORTE-01-0145-FEDER-000003), supported by Norte Portugal Regional Operational Programme (NORTE 2020), under the PORTUGAL 2020 Partnership Agreement, through the European Regional Development Fund (ERDF) and by European Commission [project reference FP7-ENV-2013-603946].

\section{Availability of data and materials}

The datasets used and/or analysed during the current study are available from the corresponding author on reasonable request.

\section{Ethics approval and consent to participate}

All the phases of the study complied with the Ethical Principles for Medical Research Involving Human Subjects expressed in the Declaration of Helsinki. The study was approved by the University of Porto Medical School/ Centro Hospitalar São João ethics committee and all parents or legal representative signed an informed consent according Helsinki.

\section{Consent for publication}

Not applicable

\section{Competing interests}

The authors declare that they have no competing interests.

\section{Author details}

${ }^{1}$ ISPUP-EPIUnit, Universidade do Porto, Rua das Taipas n 135, 4050-600 Porto, Portugal. ${ }^{2}$ Departamento de Ciências da Saúde Pública e Forenses e Educação Médica, Faculdade de Medicina, Universidade do Porto, Al. Prof. Hernâni Monteiro, 4200-319 Porto, Portugal.

Received: 27 March 2019 Accepted: 2 September 2019

\section{Published online: 11 September 2019}

\section{References}

1. Bladh M, Carstensen J, Josefsson A, Finnstrom O, Sydsjo G. Hospitalization in adolescence and young adulthood among twins and singletons: a Swedish cohort study of subjects born between 1973 and 1983. Twin Res Hum Genet. 2013;16(3):707-15.

2. Hall JG. Twinning. Lancet. 2003;362(9385):735-43.

3. Gielen M, van Beijsterveldt CE, Derom C, Vlietinck R, Nijhuis JG, Zeegers MP, Boomsma DI. Secular trends in gestational age and birthweight in twins. Hum Reprod. 2010;25(9):2346-53.

4. Petit N, Cammu H, Martens G, Papiernik E. Perinatal outcome of twins compared to singletons of the same gestational age: a case-control study. Twin Res Hum Genet. 2011;14(1):88-93.

5. Barker DJ, Eriksson JG, Forsen T, Osmond C. Fetal origins of adult disease: strength of effects and biological basis. Int J Epidemiol. 2002;31(6):1235-9.

6. Wells JC, Chomtho S, Fewtrell MS. Programming of body composition by early growth and nutrition. Proc Nutr Soc. 2007:66(3):423-34.

7. Fonseca MJ, Severo M, Correia S, Santos AC. Effect of birth weight and weight change during the first $96 \mathrm{~h}$ of life on childhood body composition--path analysis. Int J Obes. 2015;39(4):579-85.

8. Huang RC, Burke V, Newnham JP, Stanley FJ, Kendall GE, Landau LI, Oddy WH, Blake KV, Palmer $L$, Beilin LJ. Perinatal and childhood origins of cardiovascular disease. Int J Obes. 2007:31(2):236-44.

9. Joseph KS, Fahey J, Platt RW, Liston RM, Lee SK, Sauve R, Liu S, Allen AC, Kramer MS. An outcome-based approach for the creation of fetal growth standards: do singletons and twins need separate standards? Am J Epidemiol. 2009;169(5):616-24.

10. Buekens P. Wilcox A. Why do small twins have a lower mortality rate than small singletons? Am J Obstet Gynecol. 1993;168(3 Pt 1):937-41.

11. Buckler JM, Green M. The growth of twins between the ages of 2 and 9 years. Ann Hum Biol. 2008;35(1):75-92.
12. van Dommelen $P$, de Gunst $M$, van der Vaart $A$, van Buuren $S$, Boomsma D. Growth references for height, weight and body mass index of twins aged 0-2.5 years. Acta Paediatr. 2008;97(8):1099-104.

13. Segal NL. Twins' injuries: genetic and environmental risks / twin research reports / human interest stories. Twin Res Hum Genet. 2011;14(2):213-6.

14. Larsen PS, Kamper-Jorgensen M, Adamson A, Barros H, Bonde JP, Brescianini S, Brophy S, Casas M, Charles MA, Devereux G, et al. Pregnancy and birth cohort resources in europe: a large opportunity for aetiological child health research. Paediatr Perinat Epidemiol. 2013;27(4):393-414.

15. Group WMGRS. WHO child growth standards: length/height-for-age, weight-for-age, weight-for-length, weight-for-height and body mass index-for-age: methods and development. Geneva: World Health Organization; 2006.

16. Horlick M, Arpadi SM, Bethel J, Wang J, Moye J Jr, Cuff P, Pierson RN Jr, Kotler D. Bioelectrical impedance analysis models for prediction of total body water and fat-free mass in healthy and HIV-infected children and adolescents. Am J Clin Nutr. 2002;76(5):991-9.

17. Schaefer F, Georgi M, Zieger A, Scharer K. Usefulness of bioelectric impedance and skinfold measurements in predicting fat-free mass derived from total body potassium in children. Pediatr Res. 1994;35(5):617-24.

18. National High Blood Pressure Education Program Working Group on High Blood Pressure in Children and Adolescents. The fourth report on the diagnosis, evaluation, and treatment of high blood pressure in children and adolescents. Pediatrics. 2004;114(2 Suppl 4th Report):555-76.

19. Kramer MS, Platt RW, Wen SW, Joseph KS, Allen A, Abrahamowicz M, Blondel B, Breart G. A new and improved population-based Canadian reference for birth weight for gestational age. Pediatrics. 2001;108(2):E35.

20. R Core Team. A language and environment for statistical computing. Vienna: R Foundation for Statistical Computing; 2016.

21. Rogers IS, Ness AR, Steer CD, Wells JC, Emmett PM, Reilly JR, Tobias J, Smith GD. Associations of size at birth and dual-energy X-ray absorptiometry measures of lean and fat mass at 9 to 10 y of age. Am J Clin Nutr. 2006 84(4):739-47.

22. Willig AL, Hanks $\sqcup$, Fernandez JR. Birth weight is associated with body composition in a multiethnic pediatric cohort. The open obesity journal. 2011;3:4-8.

23. Guo SS, Chumlea WC. Tracking of body mass index in children in relation to overweight in adulthood. Am J Clin Nutr. 1999;70(1):145S-8S.

24. Katzmarzyk PT, Perusse L, Malina RM, Bouchard C. Seven-year stability of indicators of obesity and adipose tissue distribution in the Canadian population. Am J Clin Nutr. 1999;69(6):1123-9.

25. Ashworth A, Millward DJ. Catch-up growth in children. Nutr Rev. 1986;44(5): 157-63.

26. Hediger ML, Overpeck MD, Maurer KR, Kuczmarski RJ, McGlynn A, Davis WW. Growth of infants and young children born small or large for gestational age: findings from the third National Health and nutrition examination survey. Arch Pediatr Adolesc Med. 1998;152(12):1225-31.

27. Strauss RS, Dietz WH. Growth and development of term children born with low birth weight: effects of genetic and environmental factors. J Pediatr. 1998;133(1):67-72.

28. de Geus EJ, Posthuma D, ljzerman RG, Boomsma DI. Comparing blood pressure of twins and their singleton siblings: being a twin does not affect adult blood pressure. Twin Res Hum Genet. 2001;4(5):385-91.

29. Huxley RR, Shiell AW, Law CM. The role of size at birth and postnatal catchup growth in determining systolic blood pressure: a systematic review of the literature. J Hypertens. 2000;18(7):815-31.

30. Muhle A, Muhle C, Amann K, Dotsch J, Nusken KD, Boltze J, Schneider H. No juvenile arterial hypertension in sheep multiples despite reduced nephron numbers. Pediatr Nephrol. 2010;25(9):1653-61.

\section{Publisher's Note}

Springer Nature remains neutral with regard to jurisdictional claims in published maps and institutional affiliations. 